\title{
INNER DERIVATIONS OF DIVISION RINGS AND CANONICAL JORDAN FORM OF TRIANGULAR OPERATORS
}

\author{
DRAGOMIR Ž. ĐOKOVIĆ
}

\begin{abstract}
Let $D$ be a division ring and $k$ its center. We show that a generalized canonical Jordan form exists for triangularizable matrices $A$ over $D$ which are algebraic over $k$, i.e, satisfy $f(A)=0$ for some nonzero polynomial $f$ over $k$. This canonical form is a direct sum of generalized Jordan blocks $J_{m}(\alpha, \beta)$. This block is an $m$ by $m$ matrix whose diagonal entries are equal to $\alpha$, those on the first superdiagonal are equal to $\beta$, and all other entries are equal to zero. If $\alpha$ is separable over $k$ then we can choose $\beta=1$, but in general this cannot be done.
\end{abstract}

Notation. $D$ denotes a division ring, and $k$ its center. By $\lambda$ we denote a fixed element of $D$, and by $K$ the centralizer of $\lambda$ in $D$. We set $D^{*}=D \backslash\{0\}, K^{*}=K \backslash\{0\}$ and $U=\{\lambda \alpha-\alpha \lambda: \alpha \in D\}$. The right $D$-vector space whose elements are column $n$-vectors over $D$ is denoted by $D^{n}$ and its standard basis is denoted as usual by $\left\{e_{1}, e_{2}, \ldots, e_{n}\right\}$. By $R=D[t]$ we denote the ordinary polynomial ring over $D$ in the indeterminate $t$. If $f \in k[t]$ then $(f)$ will denote the ideal of $k[t]$ generated by $f$ while $f R(=R f)$ will denote the ideal of $R$ generated by $f$. Following [7] and [3] we shall say that an $R$-module is bounded if its annihilator (also called its bound) is not the zero ideal.

For $\alpha, \beta \in D$ we denote by $D^{n}(\alpha, \beta)$ the vector space $D^{n}$ considered as a right $R$-module in which $t$ acts as a $D$-linear transformation such that $e_{1} t=e_{1} \alpha$, and $e_{i} t=e_{i} \alpha+e_{i-1} \beta$ for $1<i \leqslant n$. Clearly, if $\beta \neq 0$ this module is cyclic with generator $e_{n}$. The 1-dimensional module $D^{1}(\alpha, \beta)$ is independent of $\beta$ and will be denoted by $D(\alpha)$. The module $D^{n}(\alpha, \beta)$ has length $n$ and each factor of its Jordan-Hölder series is isomorphic to $D(\alpha)$.

$M_{n}(D)$ is the $k$-algebra of $n$ by $n$ matrices over $D$. $X \sim Y$ for $X, Y \in M_{n}(D)$ means that $X$ is similar to $Y$.

Let $J_{m}(\alpha, \beta) \in M_{m}(D)$ denote the generalized Jordan block with diagonal entries equal to $\alpha$ and those on the first superdiagonal equal to $\beta$, while all other entries are zeros. We shall write $J_{m}(\alpha)$ for the usual Jordan block $J_{m}(\alpha, 1)$.

For $\alpha \in D$ we define $[\alpha]$ to be the set $\{\beta \alpha \gamma: \beta, \gamma \in K\}$. Note that, in general, $[\alpha]$ is not closed under addition.

\footnotetext{
Received by the editors November 16, 1983 and, in revised form, February 22, 1984 and September 10, 1984.

1980 Mathematics Subject Classification. Primary 16A42, 16A72; Secondary 12E15, 16A39.

Key words and phrases. Annihilator, Jordan-Hölder series, generalized Jordan blocks, irreducible polynomial, bounded module, similarity.

${ }^{1}$ Research supported by NSERC Grant A-5285.
} 
Results and proofs. For a general treatment of the similarity problem of matrices over a division ring we refer the reader to standard references $[3,7]$ and to Cohn's paper [4]. We shall concentrate only on the case of triangularizable matrices. It is rather surprising that even this case is nontrivial. We are mainly interested in the situation when the eigenvalues are algebraic over $k$ but for a few preliminary results we do not need that hypothesis.

Lemma 1. If $\alpha \in D \backslash U, A=\left(\begin{array}{cc}\lambda & \alpha \\ 0 & \lambda\end{array}\right), x \in D^{2}$ and $A x=x \lambda$ then $x \in e_{1} K$.

Proof. Writing $x=e_{1} \beta+e_{2} \gamma$, the equation $A x=x \lambda$ implies that $\lambda \beta+\alpha \gamma=\beta \lambda$ and $\lambda \gamma=\gamma \lambda$. Thus $\gamma \in K$. If $\gamma \neq 0$ then

$$
\alpha=(\beta \lambda-\lambda \beta) \gamma^{-1}=\left(\beta \gamma^{-1}\right) \lambda-\lambda\left(\beta \gamma^{-1}\right) \in U,
$$

which is a contradiction. Hence $\gamma=0$ and consequently, $\lambda \beta=\beta \lambda$, i.e., $\beta \in K$. We have $x=e_{1} \beta \in e_{1} K$.

LeMma 2. For $\alpha \in D$ we have $\left(\begin{array}{ll}\lambda & \alpha \\ 0 & \lambda\end{array}\right) \tilde{\exists} t\left(\begin{array}{ll}\lambda & 0 \\ 0 & \lambda\end{array}\right)$ if and only if $\alpha \in U$.

Proof. Denote the first of these matrices by $A$ and the second by $B$. If $\alpha \in U$, say $\alpha=\beta \lambda-\lambda \beta$, then

$$
\left(\begin{array}{ll}
1 & \beta \\
0 & 1
\end{array}\right) \cdot\left(\begin{array}{ll}
\lambda & 0 \\
0 & \lambda
\end{array}\right) \cdot\left(\begin{array}{cc}
1 & -\beta \\
0 & 1
\end{array}\right)=\left(\begin{array}{ll}
\lambda & \alpha \\
0 & \lambda
\end{array}\right) .
$$

If $\alpha \notin U$ then by Lemma $1, A x=x \lambda \Rightarrow x \in e_{1} K$. Since $B e_{i}=e_{i} \lambda$ for $i=1,2$ it follows that $A$ and $B$ are not similar.

Proposition 3. For $\alpha, \beta \in D$ we have $\left(\begin{array}{cc}\lambda & \alpha \\ 0 & \lambda\end{array}\right) \sim\left(\begin{array}{cc}\lambda & \beta \\ 0 & \lambda\end{array}\right)$ if and only if $U+[\alpha]=U+[\beta]$.

Proof. Denote the first of these matrices by $A$ and the second by $B$. Assume first that $A \sim B$. If one of the elements $\alpha, \beta$ belongs to $U$, then Lemma 2 implies that the other one also belongs to $U$ and so $U+[\alpha]=U=U+[\beta]$. Thus we may assume that $\alpha$ and $\beta$ are not in $U$. By hypothesis there exists $S \in \mathrm{GL}_{2}(D)$ such that $S A=B S$. Lemma 1 implies that $S e_{1}=e_{1} \gamma$ for some $\gamma \in K^{*}$. Thus $S=\left(\begin{array}{ll}\gamma & \delta \\ 0 & \varepsilon\end{array}\right)$ and the equation $S A=B S$ gives $\gamma \lambda=\lambda \gamma, \gamma \alpha+\delta \lambda=\lambda \delta+\beta \varepsilon$ and $\varepsilon \lambda=\lambda \varepsilon$. Thus $\gamma$, $\varepsilon \in K^{*}$ and $\beta=\gamma \alpha \varepsilon^{-1}+\left(\delta \varepsilon^{-1}\right) \lambda-\lambda\left(\delta \varepsilon^{-1}\right)$. Hence $U+[\beta] \subset U+[\alpha]$. The opposite inclusion follows by symmetry.

Now assume that $U+[\alpha]=U+[\beta]$. If one of the elements $\alpha, \beta$ is in $U$ so is the other and Lemma 2 implies that $A \sim B$. Thus we may assume that $\alpha$ and $\beta$ are not in $U$. By hypothesis we can write

$$
\beta=\gamma \alpha \varepsilon^{-1}+\left(\delta \varepsilon^{-1}\right) \lambda-\lambda\left(\delta \varepsilon^{-1}\right)
$$

where $\gamma, \varepsilon \in K^{*}$ and $\delta \in D$. Then

$$
\left(\begin{array}{ll}
\gamma & \delta \\
0 & \varepsilon
\end{array}\right) \cdot\left(\begin{array}{ll}
\lambda & \alpha \\
0 & \lambda
\end{array}\right)=\left(\begin{array}{ll}
\lambda & \beta \\
0 & \lambda
\end{array}\right) \cdot\left(\begin{array}{ll}
\gamma & \delta \\
0 & \varepsilon
\end{array}\right) .
$$

Let $V$ be a bounded indecomposable right $R$-module. The bound of $V$ has the form $f^{n} R$ where $f$ is an irreducible polynomial in $k[t]$. Moreover, $V$ is determined uniquely, up to isomorphism, by its bound. We shall now describe such indecomposable modules $V$ in the case when $f$ has a root in $D$. Then $f$ factorizes in $R$ into a product of linear polynomials (see [6, Corollary 4.7, p. 366]). 
THEOREM 4. Let $\lambda$ be algebraic over $k$ with minimal polynomial $f$. The module $V=D^{n}(\lambda, \mu), n \geqslant 2$, is decomposable if and only if $\mu \in U$. If $V$ is indecomposable then its bound is $f^{n} R$.

Proof. Assume that $\mu \in U$, i.e., $\mu=\lambda \alpha-\alpha \lambda$ for some $\alpha \in D$. Then

$$
\left(e_{2}-e_{1} \alpha\right) \cdot t=e_{2} t-e_{1} t \alpha=e_{2} \lambda+e_{1} \mu-e_{1} \lambda \alpha=\left(e_{2}-e_{1} \alpha\right) \lambda \text {. }
$$

Thus $e_{1} D$ and $\left(e_{2}-e_{1} \alpha\right) D$ are 1-dimensional submodules of $V$. Sincc $\lambda$ is algebraic over $k, V$ is bounded. By [7, Theorem 24, p. 46] $V$ is decomposable.

Now assume that $\mu \notin U$. Observe that $f R$ is the bound of $D(\lambda)$. Hence $f^{n} R$ annihilates $V$. In order to prove that $V$ is indecomposable it suffices to show that $f^{n} R$ is in fact the bound of $V$ (see [7, Theorem 21, p. 45]). The linear transformation of $V$ defined by $x \rightarrow x t$ has the generalized Jordan block $A=J_{n}(\lambda, \mu)$ as its matrix with respect to the standard basis of $V$. The matrix $f(A)=\left(\alpha_{i j}\right)$ satisfies $\alpha_{i j}=0$ for $i \geqslant j$ and $\alpha_{i, i+1}=\beta$ is independent of $i$ and $n$. By Lemma 2 we know that $D^{2}(\lambda, \mu)$ is indecomposable and consequently $\beta \neq 0$. It follows that $f(A)^{n-1} \neq 0$, i.e., $f^{n-1}$ does not annihilate $V$.

We shall need the following property of the inner derivation $x \rightarrow \lambda x-x \lambda$ of $D$.

LEMMA 5. If $\lambda$ is algebraic over $k$ then the equation $\lambda x-x \lambda=1$ has a solution in $D$ if and only if $\lambda$ is not separable over $k$.

Proof. Let $f$ be the minimal polynomial of $\lambda$ over $k$. Next observe that $\lambda$ is not separable over $k$ if and only if $(t-\lambda)^{2}$ divides $f$. Hence the assertion of the lemma is a consequence of a more general Lemma 8.4.3 of Cohn [5, p. 220].

REMARK 1. In the case char $k=0$ the assertion of the lemma also follows from the fact that the Weyl algebra $A_{1}$ over $k$ is simple.

REMARK 2. If $\lambda$ is algebraic over $k$ and not separable over $k$ then the equation $y \lambda=\lambda(y+1)$ also has a solution in $D$. Indeed, if $x$ satisfies $\lambda x-x \lambda=1$ then we can take $y=-x \lambda$. A special case of this result is useful in the study of cyclic division algebras (see [8, Example 3, pp. 287-288]).

Now let $V$ be a finite-dimensional right vector space over $D$. We shall say that a linear transformation $u: V \rightarrow V$ is triangular if there exists a basis of $V$ with respect to which the matrix of $u$ is upper triangular. We shall say that $u$ is algebraic over $k$ if $f(u)=0$ for some nonzero polynomial $f \in k[t]$. The next proposition shows that the algebraic triangular operators can be characterized in terms of their minimal polynomials over $k$.

Proposition 6. Let $u \in$ End $_{D}(V)$ be algebraic over $k$ with minimal polynomial $f \in k[t]$, and let $\operatorname{dim} V=n<\infty$. Then $u$ is triangular if and only if every irreducible factor of $f$ in $k[t]$ has a root in $D$.

Proof. Necessity. Let $A$ be an upper triangular matrix representing $u$ with respect to some basis. Since $u$ is algebraic over $k$, all the diagonal entries of $A$ are also algebraic over $k$. Let $g$ be the product of the minimal polynomials over $k$ of all its diagonal entries. Then $g(A)^{n}=0$. Hence $f$ divides $g^{n}$ and the necessity of our condition is proved. 
Sufficiency. We shall view $V$ as a right $R$-module in which $t$ acts as $u$. Our hypothesis implies that $f$ is a product of linear polynomials in $R$ (see [6, Corollary 4.7 , p. 366]). It follows that every elementary divisor $g$ of $u$ also has the same property. This clearly implies that all the factors of a Jordan-Hölder series of $R / g R$ are 1-dimensional. Since $V$ is isomorphic to the direct sum of $R / g R$ taken over all elementary divisors of $V$, every factor of a Jordan-Hölder series of $V$ is also 1-dimensional. Hence $u$ is triangular.

We can now easily prove the Jordan canonical form theorem for triangular operators.

THEOREM 7. Let $u$ be an algebraic triangular linear operator of an n-dimensional right $D$-vector space $V$. Then there exists a basis of $V$ with respect to which the matrix of $u$ is a direct sum of generalized Jordan blocks $J_{m}(\alpha, \beta)$ with $\beta \notin U(\alpha)=\{\alpha x-x \alpha$ : $x \in D\}$. Moreover, this normal form is unique, as in the commutative case, except that the eigenvalues $\alpha$ are determined only up to conjugacy and $\beta$ may be chosen arbitrarily subject only to the condition $\beta \notin U(\alpha)$. If $\alpha$ is separable over $k$ we may choose $\beta=1$.

Proof. All assertions except the last follow from [7, Theorem 29, p. 48], and Theorem 4 above. The last assertion follows from Lemma 5.

We conclude with the following result.

Proposition 8. Let $\lambda \in D \backslash k$ be algebraic over $k, U=\{\lambda \beta-\beta \lambda: \beta \in D\}$ and $\alpha \in D \backslash U$. Then $D=U \oplus K \alpha=U \oplus \alpha K$.

Proof. By a result of Artin and Whaples [2, Theorem 13] (see also [1, Theorem 3]) the left and right dimensions of $D$ over $K$ are finite and equal to the degree of $\lambda$ over $k$. Now both assertions follow from the fact that the inner derivation of $D$ given by $x \rightarrow \lambda x-x \lambda$ is $K$-linear for both left and right $K$-vector space structures on $D$, and its kernel $K$ has dimension 1 .

ACKNOWLEDGEMENT. I would like to thank the referee for several improvements, especially in Lemma 5 and Proposition 8, and for supplying the important references [1, 2 and 5].

\section{REFERENCES}

1. A. S. Amitsur, A generalization of a theorem on linear differential equations, Bull. Amer. Math. Soc. 54 (1948), 937-941.

2. E. Artin and G. Whaples, The theory of simple rings, Amer. J. Math. 65 (1943), 87-107.

3. P. M. Cohn, Free rings and their relations, Academic Press, New York, 1971.

4.

5. _ Skew-field constructions, Cambridge University Press, Cambridge, 1977.

6. J. Dauns, A concrete approach to division rings, Helderman-Verlag, Berlin, 1982.

7. N. Jacobson, The theory of rings, Math. Surveys, no. 2, Amer. Math. Soc., Providence, R. I., 1943.

8. R. S. Pierce, Associative algebras, Springer-Verlag, New York, 1980. $3 G 1$

Department of Pure Mathematics, University of Waterloo, Waterloo, Ontario, Canada N2L 